\title{
The Study of Evolution Paths \& Causes of Bilateral Market's Cyber Effect
}

\author{
Yong Zhu \\ School of Economics \& Management \\ Chuxiong Normal University \\ Chuxiong, China \\ zhuyongbest@126.com
}

\begin{abstract}
The bilateral market is the most basic and typical cyber platform model, which lays the foundation for the more complex multilateral market research. After introducing the basic of bilateral market, the evolution paths of bilateral market's cyber effect are found out by analysis of relative theory, including positive same-side effect, negative same-side effect, positive cross-side effect and negative cross-side effect. Additionally, the causes of cyber effect are summarized, says, the change of discourse rights derived from the game of each side of cyber platform and partial resource allocation by cyber platform. The main achievements of the paper are mapping out the evolution paths of $\mathbf{4}$ kinds of cyber effects of bilateral markets, which could be easy for readers to understand and study deeply.
\end{abstract}

Keywords-bilateral market; the evolution path; cyber effect; same-side effect; cross-side effect

\section{INTRODUCTION}

According to INTERNET TRENDS REPORT 2017, the number of netizen from all over the world is up to 3.4 billion, with a high penetration rate $46 \%$, whose related 2016 business data are as follow. The shipments of global intelligent mobile phone continued increasing, with the value of $3 \%$. The global network advertising cost increased to $\$ 37$ billion, with an increase of $22 \%$. The growth of mobile advertising has behaved better than PC. The number of logistics parcel grew rapidly because of fast development of e-commerce with $9 \%$. Mobile games infiltrated into people's life. Game player's daily online time increased by 33\% (2015.07 2017.03). Electronic game grew $40 \%$, which may be up to 1.61 times per month. The cost of global cloud infrastructure was as high as \$36 billion, accounting for 37\% IT infrastructure spending. Chinese netizen spent their 55\% spare time in online entertainment, indicating that their online time exceeded TV time, and they would like to pay some money for online courses, video, live broadcast and video games etc.

Thus, the internet has penetrated into people's lives and made some change. Meanwhile, as the supporter of internet influencing the way of people's life and entertainment, cyber platform has attracted much people's attention, which means the analysis of cyber effects of bilateral market, could be capable of laying the foundation for deep study of multilateral market, and perfect the structure of theoretical cyber marketing knowledge.

\section{The CurRent RESEARCH StATUS}

Cyber platform is a cooperative and transactive cyber situation for participants and clients, being consisted of software and hardware, such as Jingdong (online shopping platform), Lily net (marriage service platform), Changjiang Securities (stock exchange platform), and Pepper (live broadcast platform). Platform model is a strategy to build a business ecosystem shared by multiple agents, and to create network effects to achieve win-win situation of multi agents. Shenjin Wang (2014) thinks that the essence of platform model is a kind of commercial value logic, and the value logic has its particularity[1]. Limin Huang (2007) thinks that when the cyber effects of a product or both sides between supply and demand attract relative businesses to come to the platform, the platform's revenue $\left(\mathrm{P}=\mathrm{P}_{1}+\mathrm{P}_{2}\right)$ from both sides $\left(\mathrm{P}_{1}, \mathrm{P}_{2}\right)$ is fixed, and both $\mathrm{P}_{1}$ and $\mathrm{P}_{2}$ have an impact on total demand and the transactive volume. Meanwhile, the formation of bilateral market needs the following conditions: (1) demands of bilateral market exist simultaneously, (2) platform could attract and maintain a sufficient number of bilateral customers, (3) there lies cross-cyber externalities on the platform [2]. It is acknowledged that Rolfs (1974) is the first economist who studied cyber effect. By studying telephone network, Rolfs found that consumers' utilities would go up with the increase of the number of the people who come into the system, which is the most typical cyber effect [3]. Thus, the definition could be presented as following: cyber effect depends on the number of people who use the cyber product or service when a consumer joins a network. Michael and Shapiro (1985) held the idea that cyber outer effect is the spillover influence that influence others derived from network, which cannot be internalized [4], indicating that cyber outer effect is similar to cyber effect, whereas there lies a little difference between them. The essential characteristics of the network effect derived from not some single internet companies but the whole internet industry, and the enterprise that breaks the limit of cyber scale is able to acquire the whole resources, which is called "The Winner Get All" [5]. Although the phenomena "The Winner Get All” is universally acknowledged, Fengbo Liu and Xuliang Wu (2014), by analyzing Tencent's business, concluded that the enterprise's cyber effect isn't comprehensive when the professional evaluates the market situation, because the ability of enterprises to eliminate competitors and restrict competitors' trading conditions by scale advantage, in most cases, is 
severely weakened, when consumer have many alternative platforms [6].

As the internet penetrates into people's lives and study, the research on the network effect has become more and more complete, but the thoughts on cyber effect of many scholars vary vastly, which shows that the scholars have different cognitions about network and cyber effect [7], indicating that it is of great academic significance for scholars to study cyber effect.

\section{EVOLUTION PATHS OF CYBER EFFECT}

Cyber effect of cyber platform is that the value of one taking part in the cyber platform depends on the number of platform's current users, including same-side effect and crossside effect. The basic and typical academic terms are shown in Fig.1, with a simple and basic structure map, in which group A, group B and the cyber platform are listed, where group A and group B stand for different kinds of users, such as the businesses, consumers, advertising companies etc., and the number of dots in group A or group B is the number of its users When the number of group $A$ increases, just because an unknown outer factor exerts some impact on group A, one of the 4 kinds of phenomenon may occur, 2 of which are called same-side effect when the number of group A continues changing, and the rest situation are cross-side effect when the number of group B starts to vary. The evolution paths of All 4 kinds of cyber effects are shown in Fig.2 Fig.5 respectively.

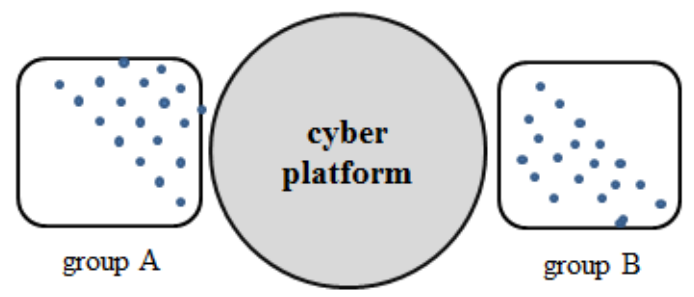

Fig. 1. The bilateral market’s structure

\section{A. Evolution Path of Positive Same-side Effect}

Positive same-side effect is defined as following: the number of group A increases for an outer factor having an impact on group $\mathrm{A}$, which leads to a greater expansion itself, and the evolution path is shown in Fig.2. During the whole process, all changes, including the change of originate situation, middle situation and final situation, are all occurred at group A. So is group B, if it could satisfy the same condition.

\section{B. Evolution Path of Negative Same-side Effect}

Negative same-side effect is that the number of group A increases for an outer factor having an impact on group A, resulting that the number of group $A$ lessens further, and the evolution path is shown in Fig.3. During the whole process, all changes, including the change of originate situation, middle situation and final situation, are all occurred at group A. Being different from positive same-side effect, negative same-side effect makes the number of group A decrease finally. So is group $\mathrm{B}$, if it could satisfy the same condition.

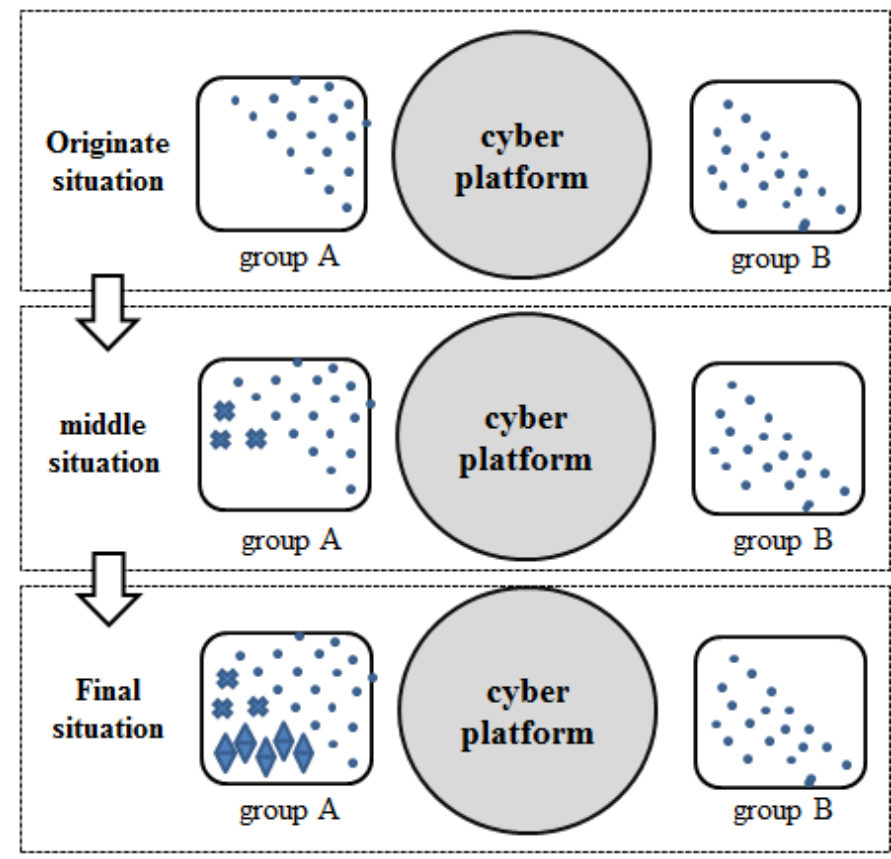

Fig. 2. The evolution path of positive same-side effect

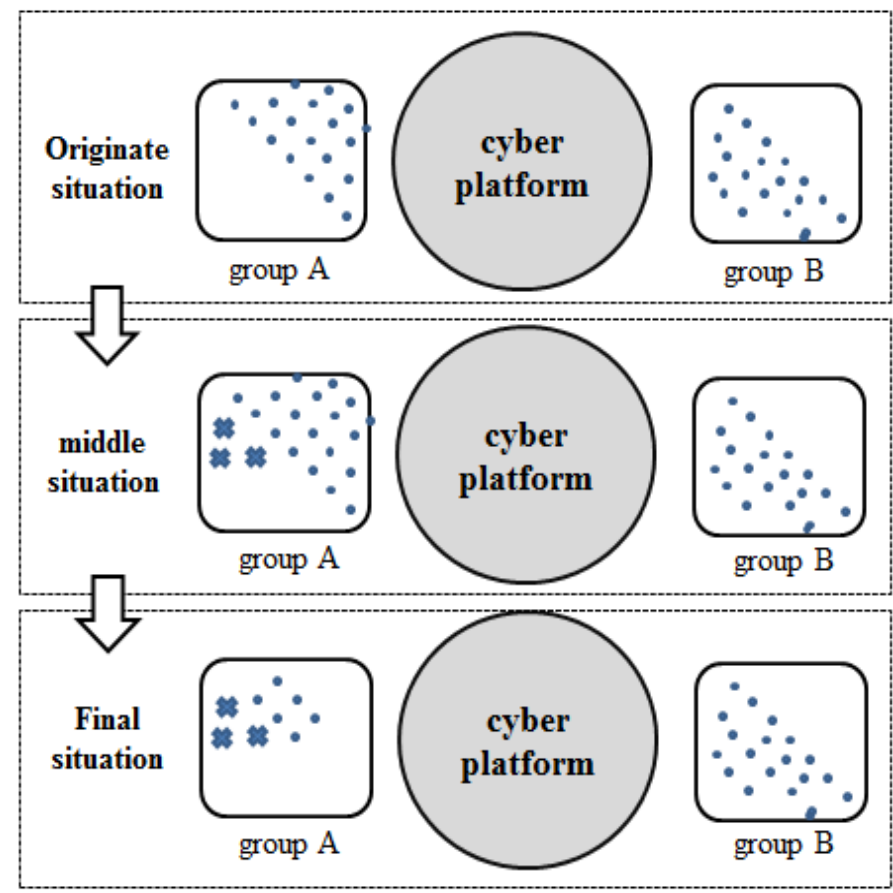

Fig. 3. The evolution path of negative same-side effect

\section{Evolution Path of Positive Cross-side Effect}

Positive cross-side effect is defined as following: the number of group A increases for an outer factor having an impact on group A, resulting in an increase of group $\mathrm{B}$, and the evolution path is shown in Fig.4. During the process, the variations of originate situation and middle situation occur at group A, while the change of final situation happens at group B. Meanwhile, the number of group B increases for an outer factor 
having an impact on group $\mathrm{B}$, resulting in an increase of group A, which is also called positive cross-side effect.

\section{Evolution Path of Negative Cross-side Effect}

Negative cross-side effect is that the number of group A increases for an outer factor having an impact on group A, resulting that the number of group $B$ lessens, and the evolution path is shown in Fig.5. During the process, the variations of originate situation and middle situation occur at group A, while the change of final situation happens at group B. Meanwhile, the number of group $B$ increases for an outer factor having an impact on group $\mathrm{B}$, resulting in a loss of group $\mathrm{A}$, which is also called negative cross-side effect.

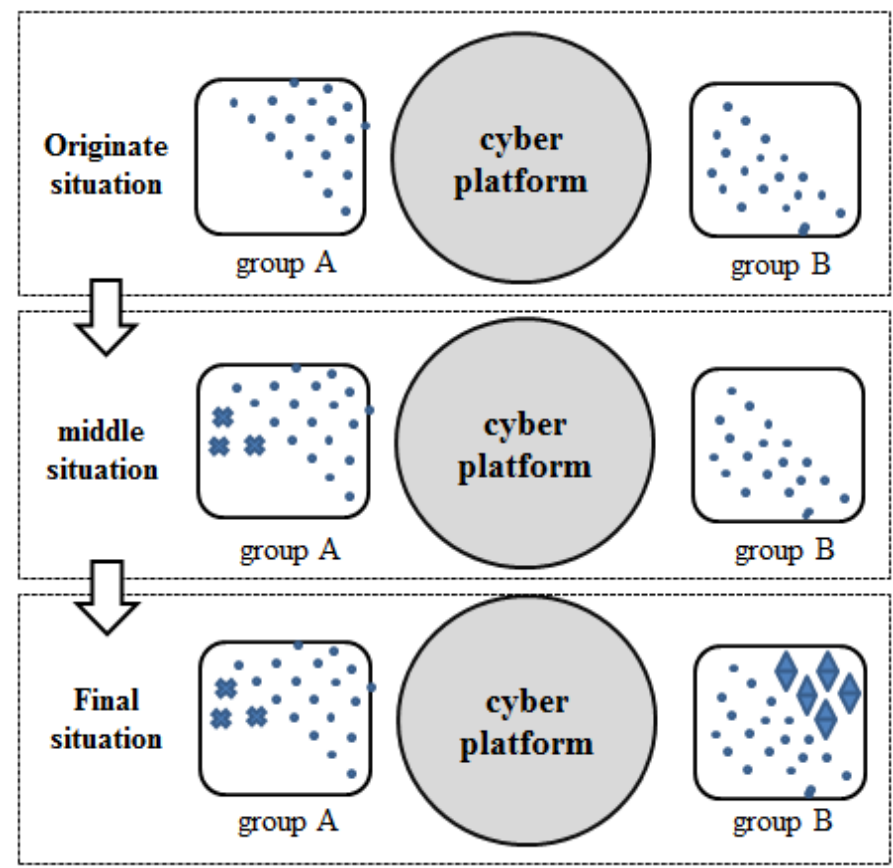

Fig. 4. The evolution path of positive cross-side effect

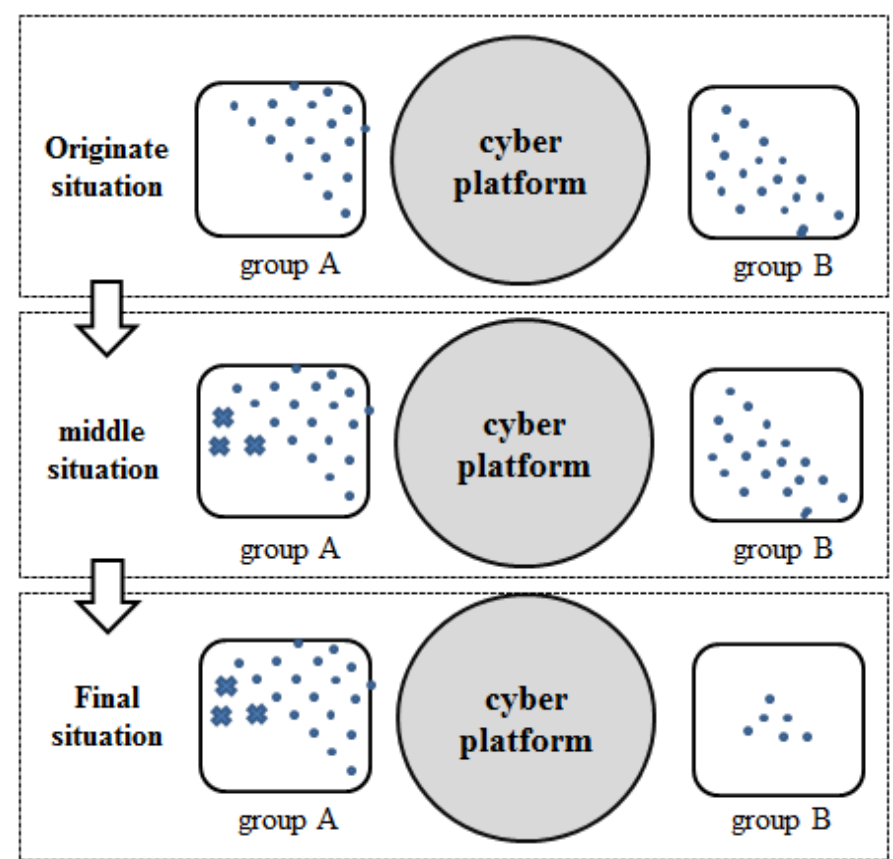

Fig. 5. The evolution path of negative cross-side effect

\section{THE CAUSES OF CYBER EFFECT}

\section{A. The Cause of Positive Same-side Effect}

When the number of group A increases for an outer factor exerting an impact on group A, group A could acquire much more discourse rights among all negotiations, including the one between group $\mathrm{A}$ and group $\mathrm{B}$, the one between group $\mathrm{A}$ and the platform and the one between group $B$ and platform, so that group A will be more attractive for users or potential users. As a result, the number of group A could increase finally.

Meanwhile, if more people join group $A$, the value of the platform will increase. As a rewarding, the platform, in return, will distribute more resources to group A than those to group B, resulting that group A's available resource could be much more than those of group B. Undoubtedly, more and more people would like to join group A, even the users of group B.

\section{B. The Cause of Negative Same-side Effect}

During a period after establishing the cyber platform successfully, there isn't any outer resource purred into the platform, says, the resources of both the platform and group A stay unchangeable. Therefore, more people pouring into group A will lessen the average available resources of group A, and group A will be less attractive at that time. Most of users or potential users, even those of group A, based on their selfinterest, will stay away from group A, inducing that the number of group A would decrease somehow.

\section{The Cause of Positive Cross-side Effect}

When the number of group A increases, whether group $\mathrm{A}$ is the subsidized or the paid, the discourse rights of both group A and the platform increase, so does the value of the platform. In order to keep the platform balanced, the platform would 
distribute more resources to group B on purpose. As a result, the group $\mathrm{B}$ will be more attractive than group $\mathrm{A}$, the number of which would increase.

On some specific occasion, we assume group A is the paid one. The total revenue of the platform will increase a lot after more people purring into the platform. In order to keep the platform healthy, the platform could spare some revenue for group $\mathrm{B}$, so that group $\mathrm{B}$ becomes more attractive and the number of group $\mathrm{B}$ increases.

\section{The Cause of Negative Cross-side Effect}

During a period after establishing the cyber platform successfully, there isn't any outer resource purred into the platform, says, the resources of the platform stay unchangeable. As more people enter into group A, group A could be more powerful in all negotiations, so that the platform distributes more resources to group A than those to group B. As a result, group B could be less attractive, the number of which may decreases finally.

Among the 4 above analyses, if the outer factor exerting some impact on group B initially, the number of group B increases somehow, affecting the number of group A or group $\mathrm{B}$ further, during which the functional principles and analyzing processes are as same as the above, so they are not presented fully here.

\section{SUMMARY}

Cyber effect of bilateral market is the basic of multilateral market, which is reflected on the change of the number of users of both groups. The cause of cyber effect of bilateral market is (1) the change of some group's discourse rights because of the number's fluctuation of its or the other group's users, and (2) partial resource allocation by cyber platform.

Therefore, much endeavor could be put into in a further research to optimize the cyber effect, only if the assignments of reallocating the discourse rights and resources partially are achieved. Due to the limited space, we don't discuss and analyze deeply.

\section{REFERENCES}

[1] Shenjin Wang. The Essence and Specialty of Platform Business Mode[J]. Commercial Research, 2014(06): 27-31. (In Chinese)

[2] Liming Huang. Two-sided markets and the Evolution of Market conformation[J]. Journal of Capital University of Economics and Business, 2007(03): 43-49. (In Chinese)

[3] Rohlfs J. A Theory of Interdependent Demand for a Communications Service[J]. Bell Journal of Economics, 1974(1):16-37.

[4] Michael K L, Shapiro C. Network Externalities, Competition and Compatibility[J]. American Economic Review, 1985(3): 424-440.

[5] Yu Fu. Competitive Strategy of Internet Service Provider in China under Network Effects[J]. Science and Technology Management, 2013(6): 192-196. (In Chinese)

[6] Fengbo Liu, Xuliang Wu. Network Effect in the Vision of Interconnection and Interworking and the Strength of Internet Enterprise[J]. Roform, 2014(11): 118-126. (In Chinese)

[7] Shuiwen Guo, Wenjing Xiao. The Study on the Interaction Mechanisms of Network Effects[J]. Economic Review, 2011(4):14-22. (In Chinese) 